\title{
Compression of the Popliteal Artery after Posterior Cruciate Ligament Reconstruction Using the Tibial Inlay Technique
}

\author{
Seung Suk Seo, MD, Jin Hyeok Seo, MD, Do Hun Kim, MD, and Byung Yoon Park, MD \\ Department of Orthopaedics, Bumin Hospital, Busan, Korea
}

\begin{abstract}
Popliteal artery compression rarely occurs after posterior cruciate ligament (PCL) reconstruction using the tibial inlay technique that allows for direct visualization of the surgical field. However, we experienced a popliteal artery compression after PCL reconstruction performed using the technique, which eventually required re-operation. Here, we report this rare case and discuss reasons of popliteal artery compression.
\end{abstract}

Keywords: Posterior cruciate ligament, Popliteal artery, Tibial inlay technique

There are several methods for posterior cruciate ligament (PCL) reconstruction. Among them, the tibial inlay technique prevents excessive bending angle of a graft tendon in the tibial tunnel, which often occurs with the conventional transtibial tunnel technique. Therefore, the tibial inlay technique is superior in prevention of graft tendon abrasion ${ }^{1,2)}$, despite the long-term clinical and radiographic outcomes of both methods do not appear to be significantly different ${ }^{3)}$.

According to the literature, PCL reconstruction with the tibial inlay technique can be associated with several complications such as hematoma, transient saphenous nerve distribution numbness, recurrent instability, laceration of the popliteal vein, failure of fixation of the bone block, and infection ${ }^{4)}$. A case of popliteal pseudoaneurysm after arthroscopic PCL reconstruction was also reported ${ }^{5)}$. However, popliteal artery compression after PCL

Received July 27, 2015; Revised October 13, 2015;

Accepted November 3, 2015

Correspondence to: Jin Hyeok Seo, MD

Department of Orthopedics, Bumin Hospital, 380-4 Deokcheon 1-dong, Buk-gu, Busan 46555, Korea

Tel: +82-51-330-3082, Fax: +82-51-330-3075

E-mail: md98hyuk@hanmail.net

This is an Open Access article distributed under the terms of the Creative Commons Attribution Non-Commercial License (http://creativecommons.org/licenses/by-nc/4.0/) which permits unrestricted non-commercial use, distribution, and reproduction in any medium, provided the original work is properly cited. reconstruction using the tibial inlay technique has rarely been reported.

Here, we present a rare case of popliteal artery compression that developed after PCL reconstruction using the tibial inlay technique for chronic rupture of the PCL.

\section{Case Report}

A fifty-year-old man was referred to our hospital for pain and instability in the left knee, which had developed after removal of the Illizarov external fixator. Twenty months ago, he suffered a midshaft open fracture (type IIIA) of the left tibia in a traffic accident. So, he underwent an open reduction and external fixation with Illizarov, which was removed after completion of bone union. Physical examination revealed no direct tenderness, swelling, and neurovascular deficit. Range of motion of the knee joint was normal (range, $0^{\circ}$ to $135^{\circ}$ ). On the knee stress test, the patient showed posterior drawer grade III instability, and there was no posterolateral rotary instability. The quadriceps active test and posterior sagging test were positive.

PCL reconstruction was performed under the final diagnosis of chronic rupture of the PCL. The femoral tunnel was created under arthroscopic guidance with the patient lying in supine position. We passed a guide wire through the tunnel for the passage of the graft tendon. Then, the operating table was tilted to the left 
side and the knee was externally rotated to partially expose the popliteal fossa. A hockey stick skin incision was made on the posteromedial aspect of the knee joint. The plane between the medial head of the gastrocnemius and the semimembranous tendons was identified. The medial head of the gastrocnemius tendon was retracted laterally to protect the neurovascular structures. Then, a vertical incision was made through the oblique popliteal ligament and posterior capsule to expose the tibial attachment site of the PCL. We used an Achilles tendon allograft: the tendinous portion of the graft was $1.1 \mathrm{~cm}$ in thickness; and tibial bony trough of the graft was $3 \mathrm{~cm}$ in length, $1.3 \mathrm{~cm}$ in width, and $1.3 \mathrm{~cm}$ in thickness. After passage of the graft, pretension of the graft was done in the femoral tunnel, and the Achilles tendon bone block was inlaid within the tibial window and fixed with a $4.5-\mathrm{mm}$ cannulated screw and a washer (DePuy Synthes, Amersfoort, Netherlands) (Fig. 1).

Immediate postoperative physical examination revealed grade I muscle power in great toe dorsiflexion, grade III muscle power in great toe plantarflexion, and numbness in the dermatome of the deep peroneal nerve. The pulse of popliteal artery was checked, but that of the posterior tibial artery and dorsalis pedis artery was not checked. The compartment pressure of the operated leg was normal. It was suspected that the decreased muscle power and numbness were due to popliteal artery compression, and emergency femoral angiography was done. The popliteal artery was found completely obstructed immediately above the knee joint (Fig. 2).

During the emergency operation, we found that the tibial bone block, not the cannulated screw and washer, compressed the popliteal artery posterolaterally (Fig. 3). With the patient placed in
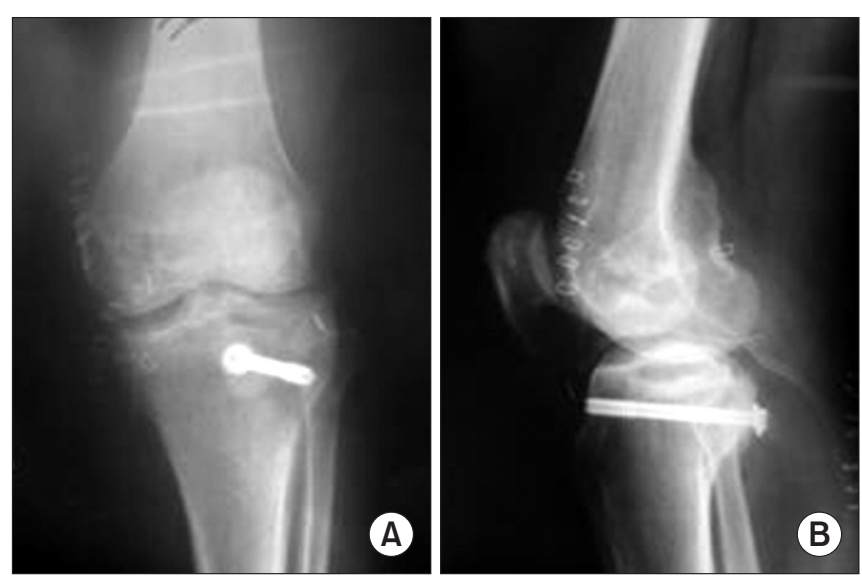

Fig. 1. Anteroposterior (A) and lateral (B) radiographs obtained after the initial posterior cruciate ligament reconstruction using the tibial inlay technique. the prone position, we made an incision along the previous operation scar and removed the screw and bone block. We reduced the thickness of the bone block from $1.3 \mathrm{~cm}$ to $1.0 \mathrm{~cm}$. We moved the bony trough more distally from the anatomic PCL footprint to secure appropriate graft tension and refixed it with a $7.0-\mathrm{mm}$ cannulated screw and a washer (DePuy Synthes) (Fig. 4). After the second operation, distal circulation was intact, and muscle weakness and numbness were resolved.

\section{Discussion}

We used the tibial inlay technique in single-bundle PCL recon-

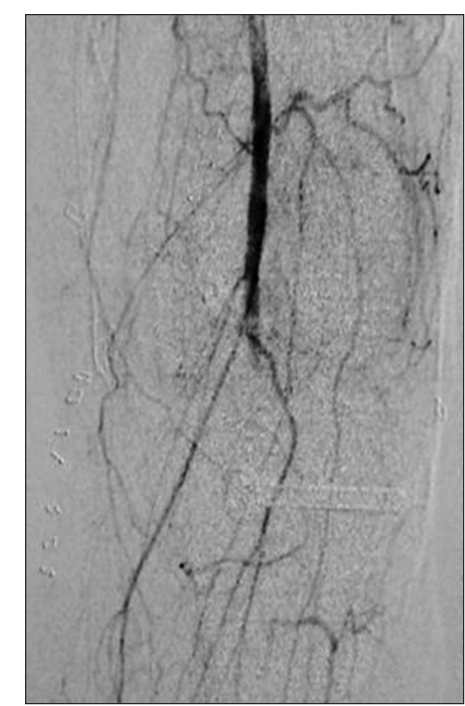

Fig. 2. Femoral angiography showing complete obstruction of the popliteal artery immediately above the knee joint.

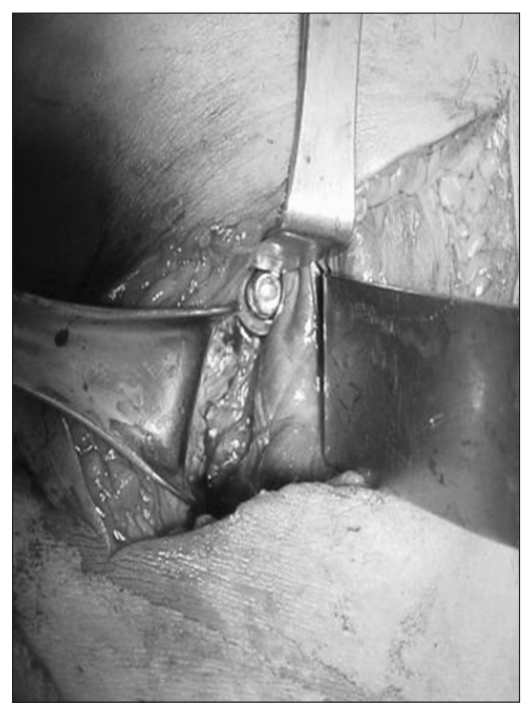

Fig. 3. Bone block compressing the popliteal artery posterolaterally. 

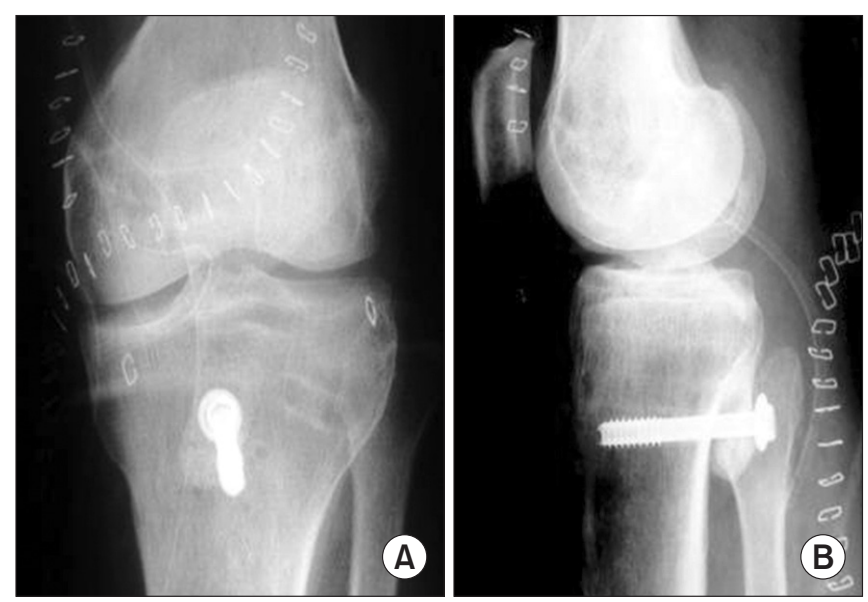

Fig. 4. After removal of the original screw and bone block, a reducedsized bone block was refixed. Anteroposterior (A) and lateral (B) radiographs obtained after the revision operation.

struction using Achilles tendon allograft because it has been reported as a favorable method providing good results ${ }^{6}$. It has been especially advocated for patients with osteopenia from disuse, fractures, and in revision surgery ${ }^{4}$. Miller et al. ${ }^{7)}$ fixed the grafted bone block with a screw on the back of the tibia after injection of barium into the femoral arteries in eight fresh-frozen knees. Then, they measured the distance from the center of the screw to the edge of the popliteal artery, the average distance of which was $21.1 \mathrm{~mm}$ (range, 18.1 to $31.7 \mathrm{~mm}$ ). They reported that the tibial inlay technique is safe with regard to the popliteal artery. Berg ${ }^{8}$ attempted anterior and posterior approaches with the injured leg in an elevated lateral position. Burks and Schaffer ${ }^{9)}$ fixed graft to the tibia using the posterior approach after passing the graft tendon through the femoral tunnel in supine position.

Originally, tibial inlay bone graft is supposed to be fixed in a prone or lateral position in the tibial inlay technique. However, we could not obtain a sufficient view of the popliteal fossa in the surgical field because the injured leg was maintained in external rotation and abduction with the patient placed in supine position. Moreover, it appeared that screw insertion for fixation of the bone block could result in lateral displacement of the bone block due to the risk of wrong placement of the screw from posteromedial to anterolateral in the supine position.

In our opinion, direct posterior approach is safer than supine position. We think that the patient should be placed in lateral or prone position when the tibial inlay technique is utilized. In our case, we used a bone block thicker than the cortical window of the tibia, and the popliteal artery compression was attributed to the short distance between the graft bone block and the posterior neurovascular structures. Therefore, it is our understanding that the bone block thickness also plays a role in the development of popliteal artery compression. In the second operation, mismatch of the tension of the graft tendon occurred. For prevention of abrasion of the graft tendon, we took care to relieve tension of the graft tendon and induce anterior displacement of the graft tendon, and the tibial bone block was re-fixed at a more distal site than the normal PCL footprint.

In general, the tibial inlay technique is a preferred method in PCL reconstruction that rarely results in neurovasulcar complication. However, graft bone block size, screw positon and direction, and patient's position during surgery may be risk factors for this complication. Therefore, the surgeon should take consideration into such factors during PCL reconstruction using the tibial inlay technique.

\section{Conflict of Interest}

No potential conflict of interest relevant to this article was reported.

\section{References}

1. Handy MH, Blessey PB, Kline AJ, Miller MD. The graft/tunnel angles in posterior cruciate ligament reconstruction: a cadaveric comparison of two techniques for femoral tunnel placement. Arthroscopy. 2005;21:711-4.

2. McAllister DR, Markolf KL, Oakes DA, Young CR, McWilliams J. A biomechanical comparison of tibial inlay and tibial tunnel posterior cruciate ligament reconstruction techniques: graft pretension and knee laxity. Am J Sports Med. 2002;30:312-7.

3. Song EK, Park HW, Ahn YS, Seon JK. Transtibial versus tibial inlay techniques for posterior cruciate ligament reconstruction: long-term follow-up study. Am J Sports Med. 2014;42:2964-71.

4. Papalia R, Osti L, Del Buono A, Denaro V, Maffulli N. Tibial inlay for posterior cruciate ligament reconstruction: a systematic review. Knee. 2010;17:264-9.

5. van Dorp KB, Breugem SJ, Driessen MJ. Popliteal pseudoaneurysm after arthroscopic posterior cruciate ligament reconstruction. Knee Surg Relat Res. 2014;26:187-9.

6. McAllister DR, Hussain SM. Tibial inlay posterior cruciate ligament reconstruction: surgical technique and results. Sports Med Arthrosc. 2010;18:249-53.

7. Miller MD, Kline AJ, Gonzales J, Beach WR. Vascular risk 
associated with a posterior approach for posterior cruciate ligament reconstruction using the tibial inlay technique. J Knee Surg. 2002;15:137-40.

8. Berg EE. Posterior cruciate ligament tibial inlay reconstruc- tion. Arthroscopy. 1995;11:69-76.

9. Burks RT, Schaffer JJ. A simplified approach to the tibial attachment of the posterior cruciate ligament. Clin Orthop Relat Res. 1990;(254):216-9. 